\title{
VIDEO-ASSISTED MINIMALLY INVASIVE CORONARY OPERATIONS WITHOUT CARDIOPULMONARY BYPASS: A MULTICENTER STUDY
}

Federico Benetti, $\mathrm{MD}^{\mathrm{a}}$

Massimo A. Mariani, MD $^{\mathrm{b}}$

Guido Sani, MD

Piet W. Boonstra, $\mathrm{MD}^{\mathrm{c}}$

Jan G. Grandjean, MD ${ }^{c}$

Pierpaolo Giomarelli, $\mathrm{MD}^{\mathrm{b}}$

Michele Toscano, $\mathrm{MD}^{\mathrm{b}}$
Objective: The need to avoid the risks associated with cardiopulmonary bypass has led to the interest in coronary operations without cardiopulmonary bypass. Patients and methods: From April 1994 to September 1995, 44 patients (mean age $63.3 \pm 10.0$ years, range 43 to 83 years) were selected for video-assisted coronary artery bypass grafting without cardiopulmonary bypass through a small anterior thoracotomy. Mean preoperative ejection fraction was $50.7 \% \pm 13.4 \%$ (range $20 \%$ to $65 \%$ ). Four patients had left ventricular dysfunction (ejection fraction below 35\%). Thirty patients had stable angina ( 26 with class 3 angina) and 14 had unstable angina. One had recurrent angina (redo). In all cases a small $(3.5$ to $11 \mathrm{~cm})$ anterior thoracotomy (43 left and one right) was performed and the harvesting of the left internal thoracic artery was video-assisted by thoracoscopy. Results: The left internal thoracic artery was used in 43 cases to graft the left anterior descending coronary artery; the right thoracic mammary was used in one case to graft the right coronary artery; the radial artery was used in one case to perform a T-graft to the first diagonal and first marginal branches. We recorded one death $(2.3 \%)$ and one case of postoperative low cardiac output syndrome $(2.3 \%)$. Perioperative myocardial infarction occurred in two cases $(4.5 \%)$. We did not record noncardiac complications (cerebrovascular complications, kidney failure, prolonged ventilatory support, or wound complications). Supraventricular and ventricular arrhythmias were never detected. Conclusion: According to our experience, videoassisted coronary bypass through a small anterior thoracotomy is a new promising technique that can be considered an alternative in most cases to angioplasty and complementary to conventional coronary operations. (J Thorac Cardiovasc Surg 1996;112:1478-84)
Since the early 1980s a new interest has arisen in $S_{\text {myocardial revascularization without cardiopul- }}$ monary bypass (CPB). Initial attempts were made by Ankeney ${ }^{1}$ and Trapp and Bisarya ${ }^{2}$ in 1975 , but their experience did not lead to a widespread use of

From the Benetti Foundation, Buenos Aires, Argentina, ${ }^{a}$ the Institute for Thoracic and Cardiovascular Surgery, University of Siena, Siena, Italy, ${ }^{b}$ and the Division of Cardiothoracic Surgery, University Hospital of Groningen, Groningen, The Netherlands. ${ }^{c}$

Received for publication Sept. 19, 1995; revisions requested Oct. 24, 1995; revisions received Feb. 5, 1996; accepted for publication March 26, 1996.

Address for reprints: Massimo A. Mariani, MD, Institute for Thoracic and Cardiovascular Surgery, University of Siena, Nuovo Policlinico "Le Scotte," Viale Bracci-53100 Siena, Italy.

Copyright $(1996$ by Mosby-Year Book, Inc.

$0022-5223 / 96 \$ 5.00+0 \quad \mathbf{1 2} / \mathbf{1} / \mathbf{7 3 9 1 9}$ the technique. After their initial efforts, the technique was developed and improved mainly by Benetti, ${ }^{3-5}$ Buffolo, ${ }^{6}$ and their colleagues. Coronary surgery is still synonymous with cardiac arrest but, despite continuous improvements in perfusion techniques, the potential for morbidity and mortality associated with $\mathrm{CPB}$ remains significant.

The need to avoid these hazards can explain the aforementioned interest in coronary operations without CPB. Furthermore, the use of video-assisted thoracoscopy for the harvesting of the left internal thoracic artery (ITA) can allow coronary operations to be done through a small anterior thoracotomy, thus reducing surgical trauma and subsequent time of hospitalization. The reduction of hospital stay, along with the reduction of the use of disposable materials, can consistently decrease the cost of medical care in patients with coronary artery disease. 
The aim of this study is to demonstrate the feasibility of video-assisted coronary surgery without $\mathrm{CPB}$ through a small thoracotomy.

\section{Patients and methods}

Patient population. From April 1994 to September 1995,44 patients (mean age $63.3 \pm 10.0$ years; range 43 to 83 years) were selected for video-assisted coronary artery bypass grafting (CABG) without $\mathrm{CPB}$ through a small anterior thoracotomy. Male/female ratio was $36: 8$. The extent of coronary artery disease and the ejection fraction are reported in Table I. Mean preoperative ejection fraction was $50.7 \% \pm 13.4 \%$ (range $20 \%$ to $65 \%$ ) and four patients had left ventricular dysfunction (ejection fraction below 35\%). The indications for operating were stable angina in 30 patients (26 had CCS class 3 angina and four had CCS class 2 angina) and unstable angina in 14 patients. In one patient the stable angina was a recurrent angina, after a previous $\mathrm{CABG}$ operation.

All patients had $\beta$-adrenergic blockers before the operation (oral atenolol or metoprolol) to help the surgeon perform the anastomosis by reducing cardiac wall motion and to reduce oxygen consumption during the brief periods of coronary occlusion. All patients gave informed consent for the operation.

Surgical decision-making (Table II). The decision to perform $\mathrm{CABG}$ without $\mathrm{CPB}$ was made after evaluation of coronary angiography along with the evaluation of each patient's risk factors. Inclusion criteria were as follows: (1) coronary artery disease involving the left coronary artery system (stenoses $>50 \%$ and good runoff) with an undiseased right coronary artery or with a right coronary artery with stenoses or occlusion not eligible for surgical treatment; (2) occlusion or stenosis of the right coronary artery not suitable for percutaneous transluminal coronary angioplasty (PTCA). The presence of extensive aortic calcification with specific contraindication to deep hypothermic circulatory arrest or femoral arterial cannulation was considered an additional factor influencing the indication for surgical treatment without CPB. Noncoronary risk factors, such as severe peripheral vessel disease, chronic obstructive pulmonary disease, or kidney failure, were also taken into consideration as additional indications by avoiding CPB in these patients. Exclusion criteria were the presence of any associated cardiac disease necessitating CPB for surgical treatment (i.e., left ventricular aneurysm or valvular disease) and three-vessel disease. The presence of an intramyocardial left anterior descending coronary artery was considered a contraindication.

Surgical technique. All patients had conventional radial artery cannulation and two central venous lines. A double-lumen endotracheal tube was used to collapse the left lung during the procedure. Anesthesia was induced and maintained with fentanyl, diazepam, pancuronium, and nitrous oxide. All patients were systematically heparinized with sodium heparin $(150 \mathrm{IU} / \mathrm{kg})$. In all cases, the CPB system was set up but not primed so that all disposable material remained sterile and could be reused, and the perfusionist was on standby.

Small thoracotomy video-assisted by thoracoscopy. A left anterior small thoracotomy was used in 43 cases to graft
Table I. Preoperative data: Video-assisted coronary surgery through a small left anterior thoracotomy

\begin{tabular}{lc}
\hline Patients & 44 \\
Mean age (yr) & $63.3 \pm 10.0 \mathrm{yr}$ \\
Male/female ratio & $36 / 8$ \\
Mean CAD extent & 1.2 \\
Mean EF (\%) & $50.7 \pm 13.4$ \\
Diabetes & 19 \\
Hypertension & 21 \\
COPD & 5 \\
Obesity & 6 \\
Renal insufficiency & 4 \\
Peripheral vascular disease & 7
\end{tabular}

$\overline{C A D \text {, Coronary artery disease; } E F \text {, ejection fraction; } C O P D \text {, chronic }}$ obstructive pulmonary disease.

the left anterior descending artery and the diagonal and marginal branches. The patients were placed in the 30degree left lateral decubitus position with the left arm above the head (Fig. 1). The small anterior thoracotomy was performed in the fifth intercostal space and the proximal left ITA was harvested with video-assisted thoracoscopy to limit the length of the incision $(3.5$ to $11 \mathrm{~cm}$ long):

In one case a right anterior small thoracotomy was performed to graft the right coronary artery with the right ITA. Whenever possible, the pleura was not opened and the ITA was dissected gently from the chest wall by means of a gauze pledget.

The thoracoscope was directed toward the left ITA (Figs. 2 and 3) through an incision made in the fifth or sixth intercostal space, along the medial axillary line. Video-assisted thoracoscopy permitted the harvesting of the proximal end of the ITA pedicle with adequate visualization even through a small thoracotomy, freeing the ITA completely by dividing the first collateral branches. The branches were occluded with clips on the ITA side, and then they were divided by means of the electrocautery. The ITA was dissected free from its origin down to two intercostal spaces below the site of the thoracotomy. This allowed for performance of the ITAcoronary anastomosis without tension and avoided any kinking of the graft. Once the lung was collapsed, the pericardium was opened anterior to the phrenic nerve (Fig. 4). The left anterior descending artery was identified by indirect vision through the pericardium or by digital inspection, after removal of the pericardial fat. The small pericardial incision was made over the artery and the cut edge of the pericardium was fixed to the chest wall with large silk stay sutures.

The radial artery was used as a T-graft, ${ }^{7,8}$ anastomosing the proximal end to the left ITA to avoid the direct aortic anastomosis and to optimize the length (Fig. 5). When the diagonal branch and the marginal branch were grafted, the pericardial incision was extended toward the phrenic nerve in a $\mathrm{T}$ shape.

After exposure of the coronary artery, a 2.5 to $3 \mathrm{~cm}$ segment of the vessel was occluded, by two looping 5-0 polypropylene sutures proximal and distal to the site of 
Table II. Indication for elective video-assisted coronary surgery without CPB through a small thoracotomy

\begin{tabular}{cc}
\hline Coronary artery disease & Surgical approach \\
\hline Isolated occlusion or stenosis of the LAD & Small left thoracotomy video-assisted with thoracoscopy \\
& (left ITA) \\
Occlusion or stenosis of the LAD and marginal & Small left thoracotomy with thoracoscopy (left ITA and T-grafts \\
and/or diagonal branches (and undiseased RCA) & with RA or IEA) \\
Occlusion or stenosis of the RCA not eligible for PTCA & Small right thoracotomy video-assisted with thoracoscopy
\end{tabular}

$C P B$, Cardiopulmonary bypass; IEA, inferior epigastric artery; ITA, internal thoracic artery; $L A D$, left anterior descending; $P T C A$, percutaneous transluminal coronary angioplasty; $R A$, radial artery; $R C A$, right coronary artery.

the anastomosis. The two stay sutures were carefully retracted while the anastomosis was being performed to partially occlude and immobilize the coronary artery. Furthermore, a blower-humidifier device blowing mixed oxygen and saline solution was used to obtain a bloodless field while the anastomosis was being done. The coronary anastomoses were performed with a running 7-0 polypropylene suture.

The heart was frequently irrigated with warm saline solution to avoid heat dispersion and the subsequent fall in core temperature and to improve regional and global wall motion, occasionally impaired by manipulation. At the end of the procedure systemic heparin was reversed with protamine.

If the pleura was not opened, a small chest tube was left in place and removed the day of the operation; otherwise a conventional large chest tube was used and was removed after 12 to 24 hours.

Hemodynamic study. Pharyngeal temperature, cardiac index, carbon dioxide production, and mixed venous blood oxygen saturation were measured after the induction of anesthesia (T0), during the coronary operation (T1), and 6 hours after the operation (T6). Cardiac index was measured by the thermodilution technique. Carbon dioxide production was measured with a Siemens 930 carbon dioxide analyzer (Siemens Corp., Union, N.J.). All data were expressed as mean \pm standard deviation.

One-way analysis of variance was used for the comparison of group means of continuous variables, that is, hemodynamic data at each interval (T0, T1, and T6). Differences were considered to be significant when the $p$ value was less than 0.05 .

Follow-up and angiographic study. Fifteen patients underwent early angiographic study 24 to 48 hours after the operation. Both native coronary circulation and grafts were investigated. The remaining 29 patients refused the study and underwent treadmill stress tests at 1 month.

This study was approved by the institutional review board and all patient gave written informed consent to the operation.

\section{Results}

Surgical data. The left ITA was used in 43 cases to revascularize the left anterior descending coronary artery. The right ITA was used in one case to revascularize the right coronary artery. The radial artery was used in one case to perform a sequential graft to the first diagonal branch and the first marginal branch. CPB was never needed during the operation to deal with hemodynamic deterioration or severe arrhythmias.

Mild hypotension occurred in seven cases during the procedure but was easily managed with volume correction, inotropic drugs (dopamine or dobutamine), and warm saline irrigation; in all cases inotropic drugs were discontinued before the end of the operation. Transient ST-segment changes were recorded in six patients during the coronary occlusion time; such changes were completely relieved at the moment of coronary reperfusion with no further sequelae. Ventricular arrhythmias never occurred during the procedure.

Clinical results. One death $(2.3 \%)$ occurred in a patient with preoperative severe left ventricular dysfunction: the patient died in the operating room of rapid and profound impairment in left ventricular function, followed by cardiac arrest unresponsive to treatment.

In two cases $(4.5 \%)$ a perioperative myocardial infarction occurred: In one case it occurred without hemodynamic deterioration. In the other case, a patient with preoperative severe left ventricular dysfunction, postoperative low cardiac output syndrome occurred, necessitating counterpulsation $(2.3 \%)$. Postoperative angiograms did not show evidence of graft occlusion or stenosis in either case. Prolonged ventilatory support (more than 24 hours) was not necessary. All patients were extubated within 12 hours after the operation, and six patients were extubated in the operating room.

Noncardiac complications such as cerebrovascular complications, kidney failure, or wound complications were not noted. Postoperative supraventricular and ventricular arrhythmias were not detected. Mean hospital stay was $3.9 \pm 1.5$ days. All clinical results are summarized in Table III.

Hemodynamic results (Table IV). A significant reduction of the mean cardiac index and carbon dioxide production was recorded at the time of the operation (T1), but the mixed venous blood oxygen 


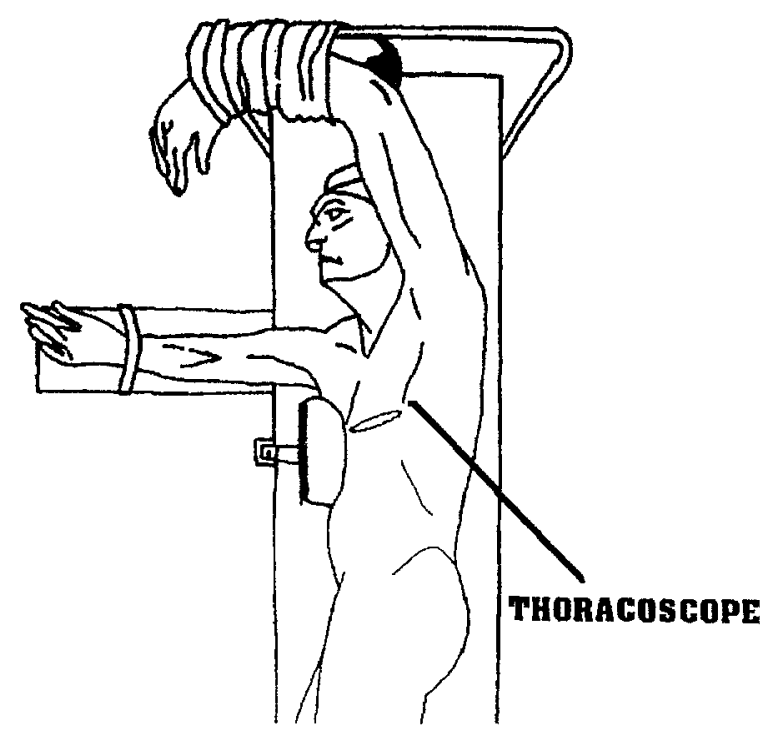

Fig. 1. The patient is positioned in the 30 - to 60 -degree left lateral decubitus position (the degrees of lateral positioning were chosen in every patient according to the shape and size of the chest). The left arm was elevated above the head. The left thoracotomy is represented at the fifth intercostal space. In the drawing the thoracoscope is inserted at the same intercostal space along the anterior axillary line.

saturation did not reach a significant drop. This was probably due to the reduction in pharyngeal temperature, despite the use of rewarming blankets and frequent irrigation with warm saline solution inside the pericardium and the pleura. The complete recovery of both metabolic and hemodynamic parameters was reached 6 hours after the operation (T6).

Angiographic results. Among 15 studied patients all grafts were patent at early evaluation. In one case a narrowing, apparently not decreasing runoff, was evident at the site of the anastomosis of a T-graft between the radial artery and the left ITA. This was probably due to the discrepancy of diameter between the two arterial grafts in that patient. In this patient the treadmill stress test was negative 1 month and 3 months after the operation. Treadmill stress tests performed 1 month after the operation showed no recurrent myocardial ischemia in any of the 29 patients who refused postoperative angiographic study.

\section{Discussion}

Currently, the main issues in "beating heart" coronary operations are the reduction of medical

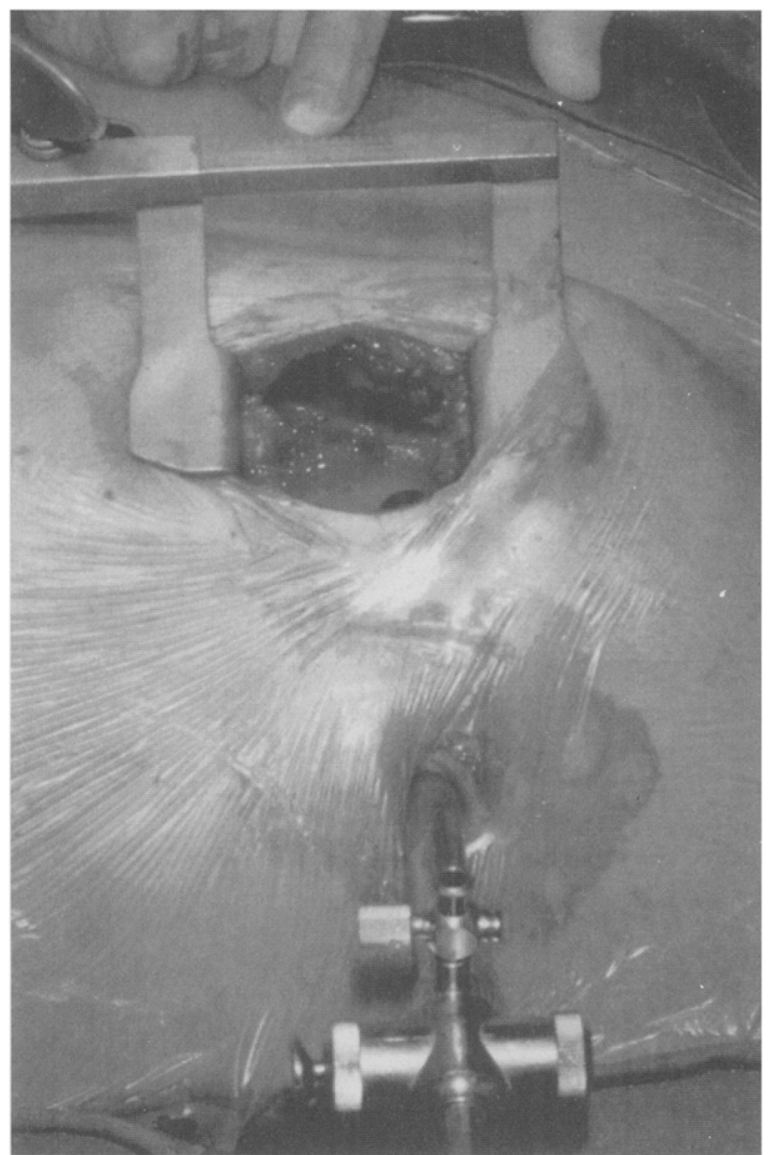

Fig. 2. The thoracoscope is inserted at the sixth intercostal space and directed toward the ITA. The distal end of the thoracoscope is visible at the lower part of the thoracotomy.

costs and the avoidance of risks associated with CPB when compared with conventional "nonbeating heart" coronary operations ${ }^{1-5,9,10}$ : in fact, there is a dramatic decrease in hospital costs owing both to the decrease of disposable materials and to the reduction in total hospital and intensive care stay. This is mainly due to a marked reduction in morbidity, which in our series fell to zero for noncardiac complications, and to a faster postoperative recovery.

In some cases there is a certain overlap in the indications for PTCA compared with our technique, but, as previously demonstrated, ${ }^{4,5} \mathrm{CABG}$ without $\mathrm{CPB}$ improves late results, thus reducing the need for further intervention. Therefore video-assisted thoracoscopic surgery seems to be cost-effective when compared with PTCA. This translates into an 


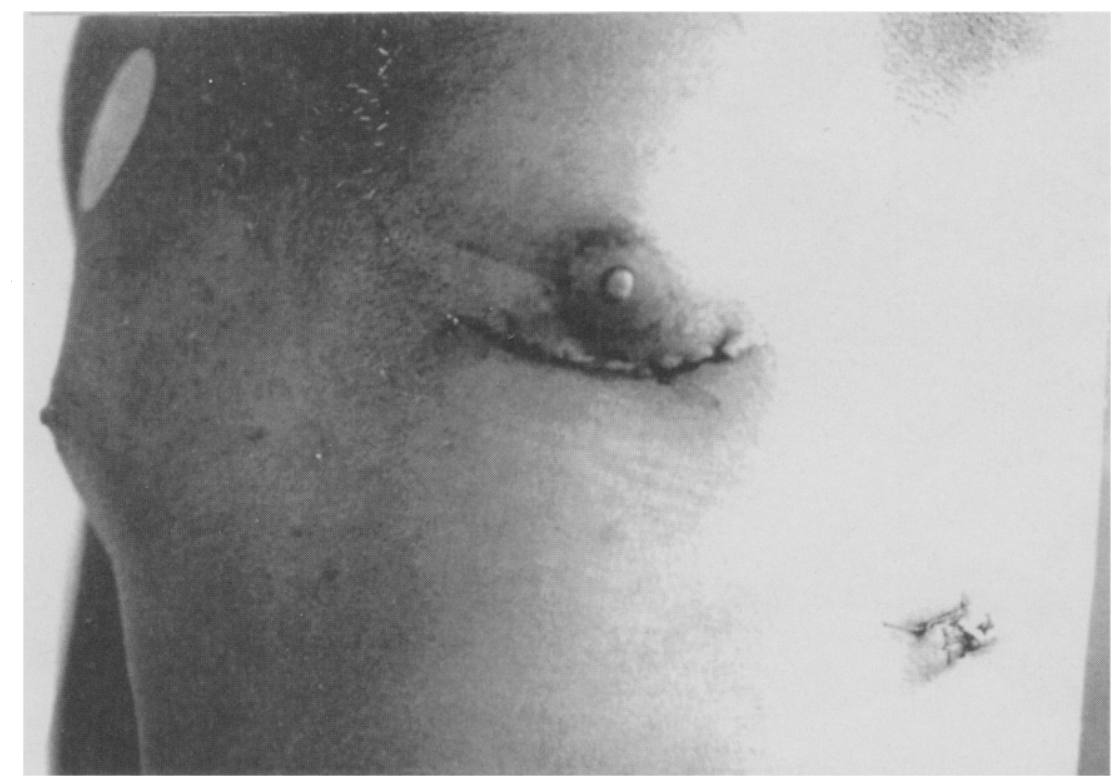

Fig. 3. A final view of the surgical access at the end of the operation. The submammary incision is $6 \mathrm{~cm}$ long. The hole where the thoracoscope was inserted is in the lower right corner.

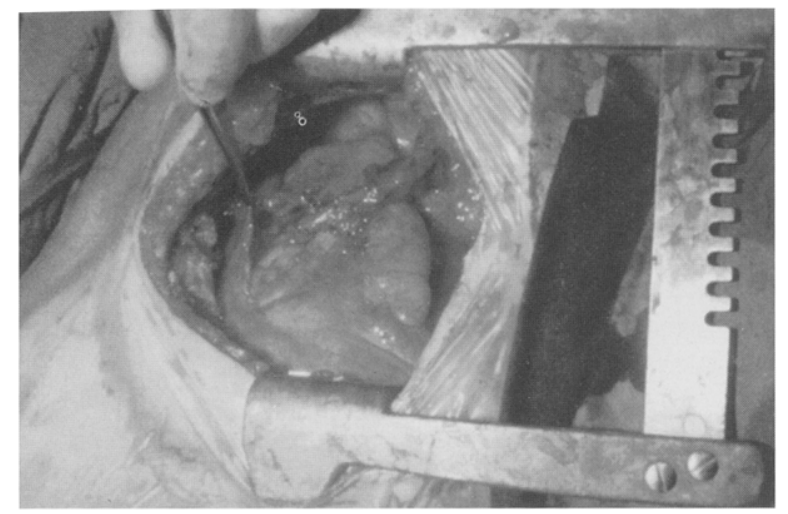

Fig. 4. The left ITA, coming from the right, is directed toward the left and anastomosed to the left anterior descending artery. The forceps grasp the superior margin of the small opening in the pericardium.

interesting alternative to PTCA in increasing numbers of patients.

However, there still remain some concerns about this technique, in particular with respect to the correct selection of cases. The main concern is whether the technique can be considered safe or not, in an era when coronary surgery yields wellestablished results. ${ }^{11}$ The experience with PTCA has clearly demonstrated that most patients can tolerate brief periods of coronary occlusion; thus the risk of myocardial damage during surgical coronary occlusion time seems acceptable.

Some authors ${ }^{12-14}$ have previously suggested a left thoracotomy approach for patients at the second coronary operation, but this point of view is still strongly controversial. ${ }^{15}$ The combined use of arterial T-grafts ${ }^{7,8}$ with video-assisted coronary surgery through a small anterior thoracotomy, moreover, is useful in patients with two-vessel disease with an undiseased right coronary artery, especially in patients with extensive calcification of the ascending aorta, avoiding both a proximal aortic anastomosis and CPB. Furthermore, the use of arterial T-grafts is attractive especially in young patients with twovessel disease and an undiseased right coronary artery, by achieving complete arterial revascularization. In addition to these benefits of coronary surgery without $\mathrm{CPB}$, video-assisted thoracoscopy reduces surgical trauma, allowing the surgeon to harvest the left ITA with only a small chest wall incision. The use of a thoracoscope is the key factor in limiting the impairment induced by a large left thoracotomy, which may be tolerated less well than a midline sternotomy. This aspect is often especially important to patients because of the psychologic aspects of a midline incision, especially for women.

In our series, the small anterior thoracotomy, both right and left, has been well tolerated with little discomfort. Finally, the chance to revascularize the 


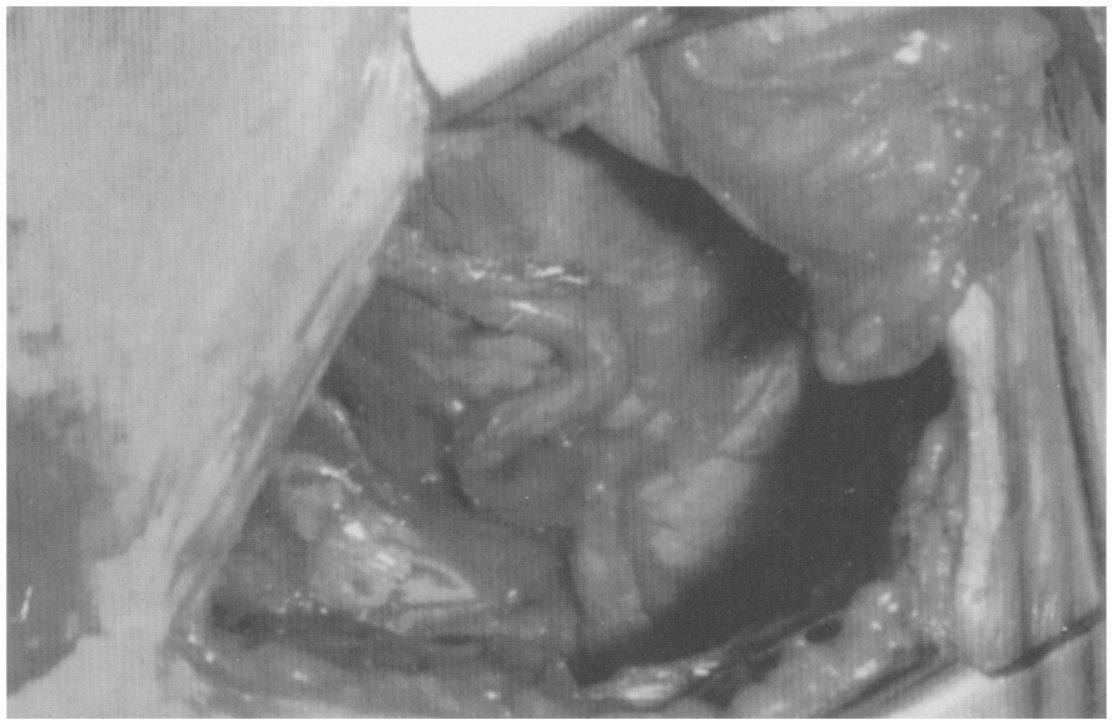

Fig. 5. Through a small left thoracotomy approach, the left ITA is directed downward to the left anterior descending artery. The proximal anastomosis (T-graft) of the radial artery is $2 \mathrm{~cm}$ before the terminolateral anastomosis with the left anterior descending artery. The radial artery graft is anastomosed side to side on the first diagonal branch and end to side on the first marginal branch.

Table III. Results: Video-assisted coronary surgery through a small thoracotomy

\begin{tabular}{lc}
\hline Patients & 44 \\
Death & $1(2.3 \%)$ \\
LCO $\Rightarrow$ IABP & $1(2.3 \%)$ \\
Mean MB percentage & $3.8 \pm 3.1$ \\
PMI & $2(4.5 \%)$ \\
Mean hospital stay (days) & $3.9 \pm 1.5$ \\
\hline
\end{tabular}

$M B$ percentage, MB-CK over total creatine kinase ratio; $L C O \Rightarrow I A B P$, postoperative low cardiac output syndrome requiring intraaortic balloon pump; $P M I$, perioperative myocardial infarction.

right coronary artery, in its first and midportions, with the right ITA via a small right anterior thoracotomy may expand the number of cases eligible for this kind of operation. In particular, undergoing a second CABG with the need of isolated revascularization of the right coronary artery or patients with isolated disease of the right coronary artery not eligible for PTCA will benefit from video-assisted minimally invasive coronary surgery, using the right ITA via the small right anterior thoracotomy.

Another advantage of this technique is that patients otherwise not eligible for coronary surgery, because of extensive aortic calcification or relative contraindication to $\mathrm{CPB}$, can benefit from coronary
Table IV. Hemodynamic results

\begin{tabular}{lcccc}
\hline & T0 & T1 & T6 & Significance $^{*}$ \\
\hline $\mathrm{t}\left({ }^{\circ} \mathrm{C}\right)$ & 36.9 & 35.2 & 37.5 & $\begin{array}{c}\text { T0 vs T1, T1 } \\
\text { vs T6 }\end{array}$ \\
$\mathrm{CI}\left(\mathrm{L} / \mathrm{min} / \mathrm{m}^{2}\right)$ & $3.5 \pm 0.9$ & $2.6 \pm 0.5$ & $4.0 \pm 1.1$ & $\begin{array}{c}\mathrm{T} 0 \text { vs T1, T1 } \\
\text { vs T6 }\end{array}$ \\
$\begin{array}{l}\mathrm{VCO}_{2} \\
(\mathrm{ml} / \mathrm{min})\end{array}$ & $225 \pm 44$ & $185 \pm 25$ & $275 \pm 50$ & $\mathrm{~T} 1$ vs T6 \\
$\mathrm{SVO}_{2}(\%)$ & $73 \pm 11$ & $70 \pm 7$ & $71 \pm 9$ & NS \\
\hline
\end{tabular}

$t$, Pharyngeal temperature; $\mathrm{Cl}$, cardiac index; $\mathrm{VCO}_{2}$, oxygen consumption; $\mathrm{SVO}_{2}$, mixed venous blood oxygen saturation.

*Differences were considered to be significant when $p$ was less than 0.05 .

surgery without the negative effects of CPB or deep hypothermic circulatory arrest. In such patients, in addition to performing coronary surgery without CPB through a standard midline sternotomy, combined left and right small thoracotomies would allow grafting the majority of coronary vessels.

As for patients with left ventricular dysfunction, a meticulous judgment in cost/benefit ratio must be made, because the risk of coronary surgery in these patients is mainly related to myocardial protection and elective cardiac arrest. If this problem is eliminated, intraoperative myocardial damage is minimized and the effects of revascularization in terms of 
regional and global wall motion recovery can be evaluated in real time. However, in those patients the manipulation of the heart could lead to more severe hemodynamic impairment than in patients with normal or moderately depressed left ventricular function, and the need to perform emergency CPB may occur. In these cases the small left thoracotomy may be quickly converted in a standard midline sternotomy by rotating the patient in the supine position and performing a conventional cannulation of the right atrium and the ascending aorta. Alternatively, an emergency femoro-femoral CPB may be performed, thus avoiding the midline sternotomy.

As for hemodynamic responses, great care must be taken during the first 6 postoperative hours, in particular when there is a reduction of body temperature. In conclusion, video-assisted coronary surgery through a small left thoracotomy is a new promising technique that can be considered an alternative to PTCA and complementary to conventional CABG. The meticulous selection of cases, along with the expanded use of the technique and long-term clinical and angiographic studies, will further clarify the relationship among these three methods of myocardial revascularization.

\section{REFERENCES}

1. Ankeney JL. To use or not to use the pump oxygenator in coronary bypass operations. Ann Thorac Surg 1975;19:108-9.

2. Trapp WG, Bisarya R. Placement of coronary artery bypass graft without pump oxygenator. Ann Thorac Surg 1975;19:1-9.

3. Benetti FJ. Direct coronary surgery with saphenous vein bypass without either cardiopulmonary bypass or cardiac arrest. J Cardiovase Surg 1985;26:217-22.

4. Benetti FJ. Coronary artery bypass without cardiopulmonary bypass versus percutaneous transluminal coronary angiogra- phy: comparison of costs. J Thorac Cardiovasc Surg 1991; 102:802-3.

5. Benetti FJ, Naselli G, Wood M, Geffner L. Direct myocardial revascularization without cardiopulmonary bypass: experience in 700 patients. Chest 1991;100:312-6.

6. Buffolo E, Gomes WI, Andrade JC, Branco JN, Maluf MA, Palma JH, et al. Myocardial revascularization without cardiopulmonary bypass: surgical results in 1090 patients. Arq Bras Cardiol 1994;62:149-53.

7. Calafiore AM, Di Gianmarco G, Luciani N, Maddestra N, Di Nardo E, Angelini R. Composite arterial conduits for a wider arterial myocardial revascularization. Ann Thorac Surg 1994; 58:185-91.

8. Tector AJ, Amudsen S, Schmal TM, Kress DC, Peter M. Total revascularization with $\mathrm{T}$ grafts. Ann Thorac Surg 1994;57:33-9.

9. Moshkovitz Y, Mohr R. Coronary artery bypass without cardiopulmonary bypass-the pros and the cons. Isr J Med Sci 1993;29:716-20.

10. Tashiro T, Todo K, Haruta $Y$, Yasunaga $H$, Nagata $M$, Nakamura M. Coronary artery bypass grafting surgery without cardiopulmonary bypass. Nippon Kyobu Geka Gakkai Zasshi 1993;41:598-602.

11. Iyer VS, Russel WJ, Leppard P, Craddock D. Mortality and morbidity after coronary artery surgery: a review of 12003 patients. Med J Aust 1993;159:166-70.

12. Kigawa I, Suma H, Marat J, et al. Reoperative coronary revascularization without cardiopulmonary bypass. Kyobu Geka 1994;47:79-82.

13. Fanning WJ, Kakos GS, Williams TE Jr. Reoperative coronary bypass grafting without cardiopulmonary bypass. Ann Thorac Surg 1993;55:486-9.

14. Kigawa I, Suma H, Nishimi M, Horii T, Fukuda S, Wanibuchi Y. The second coronary reoperation via the left thoracotomy without cardiopulmonary bypass. Nippon Kyobu Geka Gakkai Zasshi 1994;42:603-6.

15. Cosgrove DM. Is coronary reoperation without the pump an advantage? Ann Thorac Surg 1993;55:329.

16. Pfister AJ, Zaki MS, Garcia JM, et al. Coronary artery bypass without cardiopulmonary bypass. Ann Thorac Surg 1992;54: $1085-91$.

\section{Notice of correction}

In the August 1996 issue of the Journal, in the article by Taggart and associates titled "Applicability of Intermittent Global Ischemia for Repeat Coronary Artery Operations" (1996;112:501-7), an error was made. The surname of the second author was spelled incorrectly. The correct name is Carlo Aratari, MD. 